\title{
La place de l'anglais dans la chanson francophone: controverses et débat
}

\author{
Stéphanie Nutting \\ Université de Guelph \\ Canada
}

L'article qui suit présente les grandes lignes d'une communication présentée lors du 6ième colloque international sur la didactique des langues secondes tenu à l'Université McGill les 27 et 28 avril 2017 et où j'ai exposé les étapes d'une séquence pédagogique consacrée à l'exploitation de la chanson diglossique visant les apprenants du niveau B2 avancé. Plus spécifiquement, il s'agit des éléments d'un module d'un cours de 3e année offert dans une université anglophone canadienne où la majorité des étudiants avait pour langue maternelle l'anglais. Le module en question reposait alors sur le thème du choix de langue - anglais ou français? - dans le domaine de la musique populaire.

Quand nous levons le voile sur la coexistence de l'anglais et du français, nous exposons du même coup les multiples rapports de pouvoir qui sous-tendent cette coexistence. Prenons, par exemple, le quotidien même des professeurs canadiens de français langue seconde qui contemplent parfois d'un œil demi triste, demi résolu, le défi bien réel qui consiste à faire adopter le français comme langue de communication privilégiée dans une salle de classe où l'on doit constamment surveiller le dérapage vers l'anglais. Mais, beaucoup d'étudiants anglophones ignorent à quel point cette tension rappellent une dynamique qui existe en dehors des murs de l'école. L'anglais, qui exerce des pressions continuelles et complexes sur le français, surgit partout et sans cesse dans le quotidien des Francophones en Amérique du Nord (en fait, il ne serait pas inexact d'affirmer qu'il touche, à des degrés différents, les Francophones du monde entier). En effet, on a beau chasser cette langue rivale par la porte d'en avant; elle cherche toujours à revenir par la porte d'en arrière. Donc, au lieu de feindre que la situation en soit autrement, j'ai voulu souligner ce fait de la réalité contemporaine et, avec le concours des étudiants, le problématiser. Dans un sens, cette tension, souvent occultée mais bien évidente dans l'institution de la musique au Canada, me paraissait justement le gage parfait d'une expérience profondément authentique, et ce tant sur le plan sociopolitique que pédagogique. Elle motive l'élaboration d'une tâche actionnelle authentique (le débat en salle de classe qui rejoint un débat réel qui sévit au-delà de la classe) et qui s'appuie sur des documents acoustiques et écrits authentiques.

Certes, le sujet des chansons diglossiques est d'une grande actualité, justement parce que beaucoup d'artistes francophones contemporains font le choix de composer leurs chansons en anglais et de se produire surtout (ou uniquement) dans cette langue. Pensons à Béatrice Martin, mieux connue sous le nom de scène, Cœur de pirate qui, au fil des années avait construit un répertoire bien français. Récemment, cette auteurecompositrice-interprète a effectué un virage vers l'anglais en 2015. En effet, dix titres sur les quatorze chansons de son album Roses sont en anglais. Pensons aussi à la vedette de Christine and the Queens, Héloïse Letissier, cette danseuse-chanteuse de Nantes qui a éclaté sur la scène américaine avec «Tilted,» une adaptation anglaise de sa chanson française "Christine.» D'autres musiciens, comme les membres du groupe acadien Radio Radio ou le groupe montréalais, Loud Lary Ajust, sont connus pour leur diglossie jouissive et sans complexe. II faut dire que tout ce phénomène de code-switching embête les bailleurs de fonds (et de prix) dont le mandat est de soutenir et de reconnaitre la musique francophone. II s'agit donc d'un sujet en or pour discuter de l'espace culturel francophone, de la liberté d'expression des artistes, des pouvoirs du marché et du rôle des auteurs-compositeurs-interprètes, souvent vus comme les poètes de leur génération.

Dans le premier volet du présent article, nous exposerons les étapes de la séquence qui se résument ainsi : 1. Description du cours 2. Objectifs de la séquence sur le débat 3. Choix de chansons 4. Découverte des chansons 5. Présentation et analyse des documents de presse sur la controverse 6 . Préparation du débat et distribution des rôles 7. Déroulement du débat. Le deuxième volet, plus court, consistera à offrir des observations sur le déroulement de la séquence ainsi qu'une réflexion sur le contenu culturel du module. Nous ferons alors le bilan de la séquence et reviendrons de nouveau à la question de l'authentique.

\section{Cadre conceptuel et étapes :}

Mais de prime abord, il convient de réfléchir sur ce terme «authentique» qui est passablement ambigu puisqu'il semble s'appliquer autant à la nature des documents didactiques qu'aux types de tâches dont s'acquitte l'apprenant. Selon Jean-Paul Cuq et Isabelle Gruca, les documents authentiques seraient les documents «bruts,» à savoir les documents élaborés «par des francophones pour des francophones à des fins de 
communication.» Ce sont «des énoncés produits dans des situations réelles de communication et non en vue de l'apprentissage d'une seconde langue» (Cuq et Gruca 431-432). En revanche, pour Christian Puren, il s'agit plutôt de documents qui sont «représentatifs en langue-culture,» c'est-à-dire des documents qui permettent "d'extrapoler des connaissances assurées autant sur la langue française que sur la culture des pays francophones» (13). Heureusement, ces deux optiques ne s'excluent pas mutuellement et l'on peut dire que les chansons retenues ici cadrent avec les deux conceptions, peu importe celle que l'on privilégie. On constate aussi que ce terme désigne des messages écrits, oraux et visuels qui sont d'une multiplicité et d'une variété presque infinies.

Sur le plan des tâches actionnelles qui tombent sous le parapluie de l'authentique, je m'inspire surtout des recherches théoriques menées par Christian Puren. Ici, il est possible d'apprécier, dans l'ordre, la diversité et la complexité des tâches effectuées par l'apprenante : se préparer (écoute et découverte des chansons, comparaison des chansons, lecture des documents de presse); repérer (trouver des exemples de diglossie dans les paroles des chansons, identifier les arguments des documents de presse); analyser et interpréter (cerner les échecs ou réussites créatives de la diglossie dans les chansons, décider des meilleurs arguments fournis par les journalistes); extrapoler (expliciter les éléments représentatifs des réalités extratextuelles (Puren 12) ) et juger (expliciter ses arguments et décider de la force des arguments adversaires).

II va sans dire qu'un tel traitement didactique met l'oral au centre de l'exploitation. Mais on sait, d'après les affirmations de Cuq et Gruca, que l'oralité est un vaste champ. Encore faut-il distinguer entre «l'écrit oralisé» (informations radiophoniques ou télévisées, discours politiques et chansons, sketchs etc.) et «l'oral spontané» (conversations à vif, des interviews, des échanges quotidiens et des débats) (Cuq et Gruca 435). Avec les diverses articulations des tâches, allant du repérage à l'extrapolation et l'acte de juger, on doit passer d'un genre d'oralité à un autre. En l'occurrence, on jette un pont entre l'oral écrit et l'oral spontané.

\section{Description du cours :}

Dans la foulée de la création de nouveaux cours "culture-langue» ${ }^{1}$ en études françaises à l'University of Guelph, ce cours sur la chanson est venu remplacer un cours de littérature axé autrefois sur la poésie francophone. Ce nouveau cours, avec le libellé «FREN ${ }^{\star} 3160$,» présentait plusieurs défis dont l'envergure du sujet et l'absence de manuel publié n'en furent pas les moindres. ${ }^{2}$ Puisque les paroles sont considérées, depuis quelques décennies déjà, comme une forme légitime de poésie orale, le projet visait, dès le départ, à inciter les étudiants à explorer la richesse lyrique et la diversité sociopolitique des paroles à travers le prisme d'un grand corpus de chansons francophones couvrant un champ géographique hétéroclite (France, Québec, Ontario français, Acadie, Belgique, Sénégal, Rwanda). Présenté sous forme d'anthologie établie par la professeure, ce corpus regroupait un éventail de genres : chants folkloriques, chansons engagées, reggae, hip-hop, world music, électronique et chansons à auteur. En plus d'effectuer des analyses attentives des textes (ce que l'on appelle close readings en anglais), les étudiants étaient invités à développer une voix critique par rapport à la médiatisation des chansons et aux valeurs véhiculées par l'institution de la musique au Canada et au Québec. Bien sûr, pour ce faire, il était nécessaire de travailler la langue. Les objectifs de la séquence consacrée au débat reflètent donc tous le souci de souder les enjeux culturels aux enjeux linguistiques.

\section{Les objectifs du débat sur l'intégration de l'anglais dans les chansons francophones}

- Améliorer sa compréhension de l'oral par le biais de l'écoute de chansons

- Améliorer sa compréhension de l'écrit au moyen de la lecture de documents de presse

- Élargir ses connaissances culturelles, notamment dans le domaine de la musique populaire d'expression francophone (et/ou hétérolingue)

- Approfondir son vocabulaire critique par le truchement de l'argumentation

- Se sensibiliser aux codes culturels (y compris aux registres de langue) et à la dimension «vivante» d'une langue en situation minoritaire

- Améliorer sa production orale par le truchement du débat

\section{Choix de chansons}

Pour préparer et alimenter le débat, deux chansons contemporaines appartenant au genre hip-hop nordaméricain ont été retenues. II s'agit de «Blue Volvo» du groupe montréalais Loud Lary Ajust et de «Dekshoo» du groupe acadien Radio Radio. D'abord, les étudiants en ont fait l'écoute de manière indépendante (en 
suivant les paroles reproduites dans le cahier du cours) et ensuite de manière collective en salle de classe. Faute de transcription officielle des paroles, nous avons eu recours aux transcriptions disponibles en ligne qui sont, en général, moins fiables. Transcrites par des amateurs bénévoles et anonymes, ces textes comportaient souvent des transpositions erronées puisqu'elles avaient été faites «à l'oreille.» D'une certaine manière, ceci a mis en évidence la nature fondamentalement orale de ces chansons. En effet, c'était frappant de voir à quel point ni leur existence ni leur diffusion n'étaient soumises aux contraintes habituelles qui règlent la dissémination de la littérature proprement dite.

Par ailleurs, il n'est pas sans intérêt de noter ici le registre des deux chansons, surtout que la chanson «Blue Volvo" de Loud Lary Ajust ait reçu la mention «explicite» pour la teneur de propos jugés susceptibles d'offenser certains auditeurs. La chanson fait référence à la drogue de manière ostentatoire et détaillée, de même qu'elle comporte des références à caractère sexuel et violent, notamment une référence au vol d'automobile. Contrairement à Loud Lary Ajust, Radio Radio présente un monde plus bienséant. Bien que la chanson appartienne, elle aussi, au monde du hip-hop, elle ne comporte ni blasphèmes ni références à caractère sexuel ni violence. II n'y a pas, non plus, de référence explicite à la drogue. Néanmoins, la chanson demeure joliment subversive. Avec son ton ludique et désinvolte, elle critique les symptômes d'un consumérisme débridé et d'un atomisme contemporain. Les deux chansons évoquent ainsi un malaise qui est susceptible de résonner chez un public de jeunes adultes dans la vingtaine. Évidemment, la sélection des chansons est informée par plusieurs facteurs dont, bien sûr, l'âge des apprenants et le contexte de l'apprentissage. Dans le cas échéant, il a été profitable de chercher des textes passablement audacieux.

Une des raisons qui a motivé le choix de «Blue Volvo,» outre sa réussite auprès d'un public mondial, c'est la controverse dont le groupe fut l'objet lors de la remise du prix de l'Association québécoise de l'industrie du disque, du spectacle et de la vidéo (ADISQ) en 2015 (pour l'année 2014). Dans son blogue publié sur le site de Radio Canada quelques mois avant le fameux Gala, le journaliste Philippe Rezzonico cite les règlements de l'ADISQ portant sur la langue : «Ici, la langue d'expression et les chiffres sont liés. Dans la règlementation de l'ADISQ, un "produit de langue française" est un produit "dont au moins $70 \%$ du contenu est de langue française." Visiblement, selon les calculs de l'ADISQ, ce n'est pas le cas de Blue Volvo."

L'année suivante, l'Association a récidivé en excluant de la compétition un autre groupe vedette de hip-hop, le Dead Obies, pour la même raison. Cette décision a jeté de l'huile sur le feu, faisant ainsi exploser la couverture médiatique. Si je n'ai pas retenu de chanson de ce groupe, c'était pour la simple raison que ses paroles me semblaient plus crues que celles de «Blue Volvo» de Loud Lary Ajust. Par contre, j'ai retenu, pour la préparation du débat, la couverture médiatique consacrée au Dead Obies puisqu'elle nous donnait accès au point de vue des artistes qui endossent le choix du franglais. L'exemple de Loud Lary Ajust montre surtout à quel point l'institution de la musique francophone est polarisée face à l'usage de l'anglais chez les groupes émergents.

Quant à «Dekshoo» de Radio Radio, cette chanson fournit l'exemple d'une trajectoire relativement sans bémol, pour ainsi dire, ou en tout cas dépourvue de polémique. Toutefois, bien qu'elle ne soit pas associée aux mêmes controverses, son entre-deux linguistique montre à quel point la communauté musicale acadienne est à l'aise avec la diglossie. Avec «Dekshoo,» on assiste une modulation inédite qui glisse avec bonheur entre le français, l'anglais et le chiac. Soit dit en passant que la graphie fantaisiste du mot anglais «Deck shoe" (chaussure de mer) ne semble pas avoir heurté beaucoup de gens. Au contraire, il traduit une insouciance plus ou moins créative à l'égard de l'anglais normatif.

À la lumière de ces deux phénomènes de réception, deux articles de presse furent donc retenus : un premier qui défendait la décision de l'ADISQ d'exclure le Dead Obies, favorisant ainsi le français au sein de l'institution, ensuite un deuxième, rédigé par un membre de ce groupe, Yes McCan (Jean-François Ruel) qui l'a dénoncée en criant à la censure.

\section{Découverte des chansons - modèle d'exploitation}

Dans le contexte du module en question, la «découverte» des chansons correspond au volet préparatoire qui a précédé l'étape du débat. II va peut-être sans dire que les chansons suscitaient une écoute enthousiaste chez les étudiants. Si, dans le cours en général, la démarche variait un peu en fonction des chansons et des modules en question, elle reposait néanmoins toujours sur le modèle proposé par Ophélie Tremblay et inspiré d'une démarche élaborée à l'origine par Michel Boiron. 
Cette démarche, qui facilite le travail d'observation et l'analyse, comporte trois grandes étapes : a) Découverte de la chanson b) Compréhension c) Expression. ${ }^{3}$ Pour la partie a) Découverte de la chanson, il s'agit d'écouter deux fois. Après la première fois, les étudiants répondent à des questions simples telles que "Qui chante?», «À qui s'adresse la chanson?» et «Pouvez-vous identifier les instruments principaux?» Après la deuxième écoute, on entre dans la phase b) «Compréhension» qui a pour but de cerner le travail de langue qu'effectue l'auteur (ou l'auteure) des paroles et de faire ressortir les grands champs lexicaux. II s'agit de relever des expressions figées, des mots à double sens, les mots d'un registre particulier (par exemple, les mots du joual), et les références extralinguistiques. Ce dernier domaine est particulièrement important pour la compréhension de chansons comme celles du hip-hop où le texte est souvent aussi dense que le débit rapide.

La partie compréhension est nourrie davantage par le recours aux "Questionnaires,» c'est-à-dire une banque de questions portant respectivement sur le sens et le contenu, sur la forme et sur la musique et l'interprétation. Par exemple, pour travailler le sens et le contenu, la question suivante sert à susciter une compréhension par étape : «En un mot ou une phrase, résumez le contenu (l'idée) exprimé dans chacun des couplets.» Dans le contexte de notre répertoire, les références extralinguistiques comportent d'importants intertextes avec le rap américain, le consumérisme, les références particulières à la géographie urbaine, les clichés du genre, et la création de «personnages» qui s'expriment à travers ce nous appelons, faute de mieux, «l'autofiction» de la chanson.

Pour travailler la forme, l'on pourrait songer à une question comme : "Comment s'organisent les couplets et les refrains?» ou encore à "Quelles sonorités sont privilégiées?» et enfin, pour travailler la musique et l'interprétation, il est possible d'attirer le focus sur le lien qui existe entre les instruments musicaux et l'ambiance de la chanson : "Quelle ambiance la musique permet-elle de créer? (calme, agitée, joyeuse etc.)» ou encore, «quelles assonances, quelles répétitions y sont privilégiées?»

Ensuite, l'étape de la Compréhension (la deuxième dans le modèle de Tremblay) fut prolongée et adaptée afin de favoriser la comparaison dont la consigne est reproduite ici :

\section{Découverte des chansons}

1. Écoutez «Blue Volvo» de Loud Lary Ajust

- Quelles références intertextuelles avec le rap américain pouvez-vous trouver?

- Quel champ lexical pouvez-vous identifier dans la chanson?

- La volvo bleue est une métaphore de quoi, au juste?

2. Écoutez «Dekshoo» de Radio Radio.

- Lisez l'article sur le chiac dans l'Encyclopédie canadienne en ligne et notez sa définition.

- Quel champ lexical pouvez-vous repérer dans la chanson?

- Comparez «Dekshoo» de Radio Radio et «Blue Volvo» de Loud Lary Ajust à l'aide du tableau suivant :

\begin{tabular}{|l|l|}
\hline $\begin{array}{l}\text { «Dekshoo» } \begin{array}{l}\text { Décrivez les «personnages.» Donnez deux traits : } \\
1 .\end{array} \\
2 .\end{array}$ & $\begin{array}{l}\text { ¿écrivez les «personnages.» Donnez deux traits : } \\
1 .\end{array}$ \\
\hline $\begin{array}{l}\text { Repérez deux exemples du chiac } \\
1 .\end{array}$ & $\begin{array}{l}\text { Repérez deux exemples du joual } \\
1 .\end{array}$ \\
2. & 2. \\
\hline $\begin{array}{l}\text { Faites un résumé des deux premiers couplets } \\
1 .\end{array}$ & $\begin{array}{l}\text { Faites un résumé des deux premiers couplets } \\
1 .\end{array}$ \\
& \\
\hline 2. & 2. \\
\hline
\end{tabular}


Mais, c'est la troisième étape, celle de l'expression, qui correspond à la finalité du projet. Si, dans le cas d'Ophélie Tremblay, l'expression prévue relève de la création de chansons par les apprenants - et c'est certainement une excellente activité à proposer - ici l'expression prend la forme d'un débat oral actualisé en équipes et devant un jury. Le travail d'observation, d'analyse et de lecture préparatoire, permet de réinvestir ses connaissances dans la production d'un argument, mais aussi dans l'improvisation sur-le-champ.

\section{Présentation et analyse des documents de presse; exercice de pré-débat}

Comme exercice de pré-débat, les étudiants ont lu les extraits suivants en classe :

A. «Que le métissage linguistique soit un outil littéraire et enrichisse la langue, qui s'en plaindra ? II en a toujours été ainsi, de Montaigne à Antonine Maillet. Mais Dead Obies n'intègre pas des mots anglais au français comme l'ont fait avec brio Plume Latraverse ou Luc Plamondon. Dans la langue de Dead Obies, c'est plutôt le français qui se noie dans la langue dominante. Que ce créole soit de plus revendiqué politiquement comme une langue à promouvoir dans un contexte où l'anglais est déjà la langue hégémonique, c'est, oui... un suicide. Les chanteurs de Dead Obies semblent d'ailleurs le confesser lorsqu'ils hurlent :

«Do or die, j'rap un suicide.» (Christian Rioux, « J'rape un suicide...» Je souligne.)

B. «Ça prend $51 \%$ de contenu anglophone pour être subventionné en anglais et $70 \%$ de contenu francophone pour être subventionné en français. Si tu entres dans la zone grise, tu n'as droit à rien, ni à l'ADISQ* ni ailleurs. Quand tu constates tout ce qui est subventionné par l'État, du TV Hebdo aux chanteurs les plus populaires, tu te demandes pourquoi pas toi. Alors l'État est un peu en studio avec Dead Obies, en train de dicter ce qu'on a le droit et pas le droit de chanter. C'est une forme de censure. Ce sont des mesures que l'on a prises pour protéger notre culture qui deviennent un peu des barreaux. [...]» (Jean-François Ruel (Yes McCan) cité par Marc Cassivi dans "Yes McCan : en franglais svp!» Je souligne.)

*Association québécoise de l'industrie du disque, du spectacle et de la vidéo

\section{Préparation du débat et distribution des rôles}

Comme devoir, les étudiants devaient préparer le débat en lisant les articles entiers en ligne et en faisant une synthèse des deux prises de positions contraires selon la consigne suivante :

\section{Exercice de pré-débat (traitement didactique des articles de presse)}

A. Lisez les deux articles suivants :

Rioux, Christian. «J'rape un suicide...» Le devoir, le 18 juillet 2014. Sans page. Internet.

ET

Cassivi, Marc. «Yes McCan : en franglais svp!» La presse, le 14 juin 2015. Sans page. Internet.

B. Préparez le débat. Écrivez :

1. En un paragraphe, et dans vos propres mots*, expliquez pourquoi Rioux pense que le rap en «franglais» est «suicidaire» pour les jeunes francophones.

OU

2. En un paragraphe, et dans vos propres mots ${ }^{*}$, expliquez comment Yes McCan défend son groupe et leur manière de chanter dans deux langues. (Écrivez à l'endos)

*Vous pouvez quand même citer des expressions ou mots clés des auteurs à condition de ne pas trop citer et d'utiliser des guillemets.

\section{Lexique}

Voici quelques mots de vocabulaire et des formules pour vous aider à vous exprimer lors du débat :

Le chiac

Le joual

Le franglais 
Nutting, Stéphanie. «La place de l'anglais dans la chanson francophone : controverses et débat.» Nouvelle Revue Synergies Canada, №11 (2018)

L'interlecte - le mélange des langues

La diglossie - mélange de deux systèmes linguistiques ayant chacun un statut sociopolitique différent

Prendre la parole (ex. Le rap convient aux jeunes qui veulent prendre la parole)

Véhiculer une pensée

Exprimer sa pensée

$\mathrm{X}$ sert à caractériser $\mathrm{Y}$ (ex. la métaphore des souliers sert à caractériser la précarité financière)

Défendre une position

Nuire à quelque chose ou à quelqu'un

Reprocher quelque chose ou à quelqu'un

L'individu (vie privée) versus l'artiste (vie publique)

La chanson est émaillée de (ex. la chanson est émaillée de jurons)

Les jurons

Les sacres

L'échantillonnage

Le pseudonyme

Le nom de baptême

\section{Distribution des rôles}

Pour ce qui est de l'organisation du débat, deux jours avant le débat, nous avons établi les six groupes de cinq personnes. Trois groupes devaient prendre position en faveur de la déclaration; trois groupes devaient s'y opposer. La distribution des rôles et la prise de position respective - pour ou contre - ont été établies à ce moment-là.

\section{Consigne pour le déroulement du débat :}

Temps requis : 25 minutes, minimum.

Déclaration : Le franglais dans les chansons de groupes contemporains enrichit la culture francophone.

Dans un groupe de cinq (calculé selon une présence de 30 personnes).

La professeure assignera une prise de position à votre groupe (en faveur ou contre).

Étapes :

1. Consultation en groupe (10 minutes)

- Écrire ensemble des points d'argumentation - préparer des questions à poser aux adversaires

- Prévoir des statistiques ou références pour étayer votre point

- Prévoir des exemples très précis tirés des chansons au programme

- $\quad$ Anticiper les arguments des adversaires

2. Chaque groupe choisit un.e porte-parole qui débattra la question au niveau de la classe.

3. La professeure demandera quatre autres volontaires : trois juges + une personne pour surveiller le temps.

4. Les six (environ) personnes ( 3 en faveur, 3 contre) vont ensuite venir devant la classe pour débattre la question.

5. Déroulement (12 minutes) : $1^{\mathrm{er}}$ affirmatif ( 1 minute), $1^{\mathrm{er}}$ négatif (1 minute), $2^{\mathrm{e}}$ affirmatif (renforce ce que le $1^{\text {er }}$ a dit -1 minute), $2^{\mathrm{e}}$ négatif (renforce ce que le $1^{\mathrm{er}}$ a dit -1 minute); période de discussion -1 débatteur à la fois (6 min)

6. $3^{\mathrm{e}}$ négatif (discours de réfutation- dernière chance pour convaincre) $1 \mathrm{~min}$

7. $3^{e}$ affirmatif (discours de réfutation- dernière chance pour convaincre) $1 \mathrm{~min}$ Décision du jury rendue par le président ou la présidente du jury.

\section{Observations, bilan et retour sur le terme «authentique»}

Pendant le débat, il était clair que les étudiants se libéraient spontanément des notes qu'ils avaient préparées. II y a eu une implication affective évidente dont l'intensité rappelait celle d'un match sportif opposant deux équipes adversaires. Voilà, justement, la démonstration du paradoxe du ludique : plus l'organisation est serrée, c'est-à-dire plus les paramètres sont précis, plus l'improvisation est naturelle et dynamique.

Néanmoins, comme dans toute séquence didactique actualisée pour la première fois, il y a eu des pépins. Ceux-ci relevaient majoritairement de la logistique de la séquence. Dans un cours universitaire, il faut prendre en compte le taux habituel d'absentéisme. Le fait de commencer l'organisation plusieurs jours en avance a eu 
des effets inattendus sur la structure du débat dont, surtout, un déséquilibre dans la constitution des groupes. Face aux absences et aux blancs de mémoire chez les étudiants qui ne se rappelaient pas toujours de la composition de leur groupe, il a fallu réorganiser le tout le jour du débat où nous avons fini par simplement scinder la classe en deux. Cela a nui à la qualité des échanges parce que, du coup, des étudiants qui avaient préparé leur argumentation en faveur du «pour» se trouvaient soudainement dans le camp du «contre» et vice versa.

Qui plus est, en devenant membre du jury le jour du débat, les étudiants qui avaient pris position, mentalement, dans leur préparation, ont été appelés alors à rester neutres. Voilà qui semblait difficile après coup. Bref, il aurait fallu désigner le jury dès le début afin d'éviter de biais dans les délibérations. II est conseillé de nommer alors un jury d'au moins trois personnes qui doivent lire tous les articles mais qui doivent s'abstenir de prendre position avant le jour du débat.

\section{Dans quelle mesure cette séquence est-elle authentique?}

II est clair que les chansons retenues pour le débat correspondent au critère de l'authenticité en vertu de leur état «brut,» c'est-à-dire qu'elles ne sont pas du tout «didactisées» au départ, mais aussi parce que leur représentativité culturelle permet d'extrapoler sur la culture francophone, surtout celle de l'Amérique du Nord. En effet, il ne s'agissait ni d'éviter le choc culturel des thèmes propres au genre hip-hop ni d'effectuer de censure en éliminant les aspérités de la langue ou celles des mélanges de codes.

Avant tout, je cherchais à inciter les apprenants à réfléchir sur le français non pas comme un code neutre, plus ou moins maîtrisé selon la personne, mais comme un mode de rapport à l'autre et à soi-même, un mode culturel toujours en évolution. En faisant ceci, j'espérais amener les étudiants à vivre le fait français du point de vue de l'intérieur, du point de vue d'une francophone minoritaire en Amérique du Nord.

Bref, cette authenticité, je désirais la chercher tant au niveau «ontologique» qu'au niveau du corpus et des tâches. Je souhaitais que les étudiants réagissent à une tension sociale bien réelle car l'industrie de la musique se trouve à un moment charnière : le maintien du français en tant que lingua franca de l'industrie de la musique francophone est loin d'être évident. Aujourd'hui, beaucoup de jeunes musiciens rusent avec le poids du français ressenti comme une langue de l'autorité (et celle des règlements). Les étudiants découvrent les enjeux des politiques linguistiques à travers la chanson contemporaine et sont ainsi appelés à naviguer ensemble les houles de ce grand courant... avec ou sans chaussure de mer aux pieds!

\section{Appendice A -- Choix de chansons : extraits «Blue Volvo»}

You wanna be a superstar baby, bienvenue dans le nouvel âge baby

J'nous vois tout de noir vêtu

Drop top 'train de faire les fous à Las Vegas

Run around sur le boulevard naked

Sous le regard dégoûté des neighbours

Tu t'en souviens? Damn

We had a blast that night

Cops came over un tas de plaintes du voisinage

Mon boy Gio avait la Volvo bleue

On était sept boys so on faisait la job pour deux

On chantait Kanye 808

Moi j'disais tout l'temps que j'me compare à Lil Wayne

Pis qu'le char était loué, but it wasn't

J'me souviens des conneries

J'me souviens d'la fois ou t'étais pas là pis la police est venue cogner à la porte juste pour te pogner

Was a close one

You saw me chuckin' up the deuces

Yeah j't laissé tout seul, I regret it

Toute mon adolescence j'ai vu la blue Volvo spinnin' around and round and round

Prenez place les amis

Sur les black leather seats 
Parce qu'on s'en va nulle part tonight, tonight

Y'a pas d'speed limit sur la blue Volvo

The wheels keep spinnin mais 'a tourne en rond

La ceinture autour du cou

On va rouler pour toujours, let's go [...]

Loud Lary Ajust «Blue Volvo». LLA. Les disques Audiogramme, 2014.

Transcription adaptée de Genius.com : https ://genius.com/Loud-lary-ajust-blue-volvo-lyrics

«Deckshoo»

Intro : Nice nice

Chorus (sic) : Avec mes Penny Loafers ou avec mes Deck Shoes

Ej'vas marcher sur la hey ou j'vas marcher sur la hoo $(2 x)$

Canned tuna caviar, Michelina superstar

Marcher sur la Main ou marcher sur la I don't know

Ini mini miny moe c'est qui c'qui veut du Jello ?

Si ton sport c'est du vélo

Ben come on baby hello

Saute, danse autour de la question

Marcher sur la hey Ok

J't'explique que ça icitte c'est un cirque

Jet Set dans mon minivan dors dans un cot

J'ai ma case in case que ma case work pas

Head case in case que mon style work pas

Penny Loafer Deck Shoe Garde à ça Garde à ça

Garde à ça J'ai ma

Non man moi ej work ma tan, shopping channel vendre une scam

Copy coller j'su comme damn basically so basic

Chorus (sic)

So plus broke, mieux habillé marcher sur la hey

J'ai dit plus broke, mieux habillé marcher sur la ahaa

So plus broke, mieux habillé marcher sur la hey

J'ai dit plus broke, mieux habillé marcher sur la ahaa

$[\ldots]$

Radio Radio, «Deckshoo.» Belmundo Regal, Bonsound Records, 2010.

Transcription adaptée de Genius.com : https ://genius.com/Radio-radio-dekshoo-lyrics

\section{Notes}

1Je me permets d'intervertir ici les composants du terme «langue-culture», emprunté à Christian Puren (4), afin de mettre l'accent sur la culture tout en soulignant et respectant la relation étroite que celle-ci entretient avec l'apprentissage de la langue.

2 Je tiens à exprimer ma reconnaissance à la professeure Johanne Melançon de l'Université Laurentienne pour ses précieux conseils au moment où j'élaborais le nouveau cours en question.

${ }^{3}$ Boiron, dont l'approche pédagogique publiée dans la Nouvelle Revue pédagogique vise le niveau collégial en France, propose un «espace pédagogique» centré sur trois types d'activités principales : l'observation, l'expression de l'opinion, la création. De plus, il précise que le plaisir est au cœur de son approche : «Le plaisir de l'écoute reste une priorité» (11). 
Nutting, Stéphanie. «La place de l'anglais dans la chanson francophone : controverses et débat.» Nouvelle Revue Synergies Canada, №11 (2018)

\section{Bibliographie}

Boiron, Michel. «Approches pédagogiques de la chanson contemporaine.» Nouvelle revue pédagogique Collège, no. 6, février 2007, pp. 10-15.

Cuq, Jean-Paul et Isabelle Gruca. Cours de didactique du français langue étrangère et seconde. Nouvelle édition, Presses universitaires de Grenoble, 2005.

Cassivi, Marc. «Yes McCan : en franglais svp!» La presse, 14 juin 2015, http://www.lapresse.ca/arts/festivals/francofolies/201506/13/01-4877763-yes-mccan-en-franglaissvp.php.

Cœur de Pirate (Béatrice Martin). Roses. Dare to care, 2015.

Côté, Émilie. «Entre deux tounes : Loud Lary ajust écarté du Gala de I'ADISQ.» La presse, 5 juin 2015, http://plus.lapresse.ca/screens/f90cc449-35e4-4c24-9730-b08fb56a7b3b|PgaKaWVynJtq.html.

Faubert, Danièle et Mario Chenart. Écrire une chanson. vol. 2, Les structures; la musique des mots. Québec Amérique, 2011.

Larrède, Christian. «Coeur de pirate se met à l'anglais sur son troisième album.» Les Inrockuptibles, 18 septembre 2015, http://www.lesinrocks.com/musique/critique-album/coeur-de-pirate-roses/.

Laurendeau, Paul. «Chiac.» Encyclopédie canadienne, Historica Canada, http://encyclopediecanadienne.ca/fr/article/chiac/. Accès le 11 juillet 2017.

Léger, Robert. La chanson québécoise en question. Québec Amérique, 2003.

---. Écrire une chanson. Québec Amérique, 2001.

Lemieux, Yasmina. «Débat style discussion dans la salle de classe $\left(6^{e}\right.$ à $7^{e}$ année).» (guide adapté d'un texte publié par l'Association d'élocution et des débats de la Saskatchewan) Saskatchewan Teachers' Federation, 2006, www.stf.sk.ca. Accès 26 avril 2017.

Loud Lary Ajust. «Blue Volvo.» LL,. Audiogram, 2015.

---. Paroles de «Blue Volvo.» Genius.com.

Puren, Christian. «Traitement didactique des documents authentiques et spécificités des textes littéraires : du modèle historique des tâches scolaires aux cinq logiques documentaires actuelles. » Site personnel de Christian Puren, 2012, www.christianpuren.com/mes-travaux/2012j/. Accès le 26 juin 2017.

Rezzonico, Philippe. «Ni franco, ni anglo : que fera l'ADISQ de Loud Lary Ajust?» La filière Rezzonico. RadioCanada, 5 juin 2015, 16h28. http ://blogues.radio-canada.ca/lafiliererezzonico/2015/06/05/ni-franconi-anglo-que-fera-ladisq-de-loud-lary-ajust/.

Radio Radio. «Dekshoo.» Belmundo Regal. Bonsound, 2010.

---. Paroles de «Dekshoo.» Genius.com.

Rioux, Christian. «J'rape un suicide... » Le devoir, 18 juillet 2014.

Samuelson, Kate. «The Voice of a Different Generation. » Time Magazine, 6 octobre 2016, http://time.com/4518809/heloise-letissier-next-generation-leaders/.

Tremblay, Ophélie. «La chanson pour travailler l'oral et l'écrit en classe de français.» Québec français, no.165, printemps 2012, pp. 51-53. 